Research paper

\title{
$(2+1)$-dimensional local and nonlocal reductions of the negative AKNS system: Soliton solutions
}

\author{
Metin Gürses ${ }^{\mathrm{a}}$, Aslı Pekcan ${ }^{\mathrm{b}, *}$ \\ a Department of Mathematics, Faculty of Science, Bilkent University, Ankara 06800, Turkey \\ ${ }^{\mathrm{b}}$ Department of Mathematics, Faculty of Science, Hacettepe University, Ankara 06800, Turkey
}

\section{A R T I C L E I N F O}

\section{Article history:}

Received 13 August 2018

Accepted 22 November 2018

Available online 23 November 2018

\section{Keywords:}

Ablowitz-Musslimani reduction

$(2+1)$-dimensional negative AKNS

hierarchy

Hirota bilinear method

Soliton solutions

\begin{abstract}
A B S T R A C T
We first construct a $(2+1)$-dimensional negative AKNS hierarchy and then we give all possible local and (discrete) nonlocal reductions of these equations. We find Hirota bilinear forms of the negative AKNS hierarchy and give one- and two-soliton solutions. By using the soliton solutions of the negative AKNS hierarchy we find one-soliton solutions of the local and nonlocal reduced equations.
\end{abstract}

(C) 2018 Elsevier B.V. All rights reserved.

\section{Introduction}

Let $\mathcal{R}$ be the recursion operator of an integrable equation. Then the integrable hierarchy of equations are defined as

$$
v_{t_{n}}=\mathcal{R}^{n} v_{x}, \quad n=0,1,2, \ldots
$$

In [1], we proposed a system of equations

$$
\mathcal{R}\left[v_{t_{n}}-a \mathcal{R}^{n} \sigma_{0}\right]=b \sigma_{1}, \quad n=0,1,2, \ldots,
$$

where $\sigma_{0}, \sigma_{1}$ are some classical symmetries of the same integrable equation. This hierarchy represents the negative hierarchy of the integrable system defined in (1.1). For some specific choices of the constants $a, b$, and $\sigma_{0}, \sigma_{1}$ we have studied the existence of three-soliton solutions and Painlevé property of the negative KdV hierarchy where the recursion operator is $\mathcal{R}=D^{2}+8 v+4 v_{x} D^{-1}$. For this case the system of equations in (1.2) is denoted as $\operatorname{KdV}(2 \mathrm{n}+4)$ equations for $n \geq 1$. When $a=-1, b=0, n=1$, and by letting $v=u_{x}$ to get rid of nonlocal terms containing $D^{-1}$ we obtain $K d V(6)$. We have also obtained $(2+1)$-dimensional extension of this equation, the $(2+1)-\operatorname{KdV}(6)$ equation, by choosing $a=-1, b=-1, n=1$, and $\sigma_{0}=v_{x}, \sigma_{1}=v_{y}$. The expanded form of $(2+1)-\operatorname{KdV}(6)$ equation with $v=u_{x}$ is given as [2]

$$
u_{x x x t}+u_{x x x x x x}+40 u_{x x} u_{x x x}+20 u_{x} u_{x x x x}+8 u_{x} u_{x t}+120 u_{x}^{2} u_{x x}+4 u_{t} u_{x x}+u_{x y}=0 \text {. }
$$

We showed that all $(2+1)-\operatorname{KdV}(6)$ equation and $2+1$ dimensional $\operatorname{KdV}(2 n+4)$ for $n \geq 1$ possesses three-soliton solution having the same structure with the KdV equation's three-soliton solution and also Painlevé property. Negative flows have been considered earlier in [3-5].

\footnotetext{
* Corresponding author.

E-mail addresses: gurses@fen.bilkent.edu.tr (M. Gürses), aslipekcan@hacettepe.edu.tr (A. Pekcan).
} 
By using our approach (1.2), we obtain negative hierarchy of integrable equations which are nonlocal in general. Here nonlocality is due to the existence of the terms containing the operator $D^{-1}$. In the KdV case the nonlocal terms disappear by redefinition of the dynamical variable. This may not be possible for other integrable systems. A new type of nonlocal reductions of integrable systems are obtained by relating one of the dynamical variable to the time and space reflections of the other one. Such a nonlocal reduction was first introduced by Ablowitz and Musslimani [6,8]. Ablowitz-Muslimani type of nonlocal reductions attracted many researchers [10-33] to investigate new nonlocal integrable equations and find their solitonic solutions. These nonlocal integrable equations have been obtained by applying the Ablowitz and Musslimani nonlocal reductions of the AKNS [9] and other integrable systems of equations. First example was the nonlocal nonlinear Schrödinger (NLS) equation and then nonlocal modified KdV (mKdV) equation. Ablowitz and Musslimani proposed later some other nonlocal integrable equations such as reverse space-time and reverse time nonlocal NLS equation, sine-Gordon equation, $(1+1)$ - and $(2+1)$ - dimensional three-wave interaction, Davey-Stewartson equation, derivative NLS equation, ST-symmetric nonlocal complex mKdV and mKdV equations arising from symmetry reductions of general AKNS scattering problem [6-8]. They discussed Lax pairs, an infinite number of conservation laws, inverse scattering transforms and found one-soliton solutions of these equations. Ma, Shen, and Zhu showed that ST-symmetric nonlocal complex mKdV equation is gauge equivalent to a spin-like model in Ref. [24]. Ji and Zhu obtained soliton, kink, anti-kink, complexiton, breather, rogue-wave solutions, and nonlocalized solutions with singularities of ST-symmetric nonlocal mKdV equation through Darboux transformation and inverse scattering transform [25], [26]. In [27], the authors showed that many nonlocal integrable equations like Davey-Stewartson equation, T-symmetric NLS equation, nonlocal derivative NLS equation, and ST-symmetric complex mKdV equation can be converted to local integrable equations by simple variable transformations. Multidimensional nonlocal equations equations have been considered in [29-31]. Recently we studied all possible nonlocal reductions of the AKNS system. We have obtained one-, two-, and three-soliton solutions of the nonlocal NLS [32] and mKdV equations [33]. We also studied nonlocal reductions of Fordy-Kulish [34] and super integrable systems [35,36].

In this work, by the use of the formula (1.2) we obtain negative AKNS hierarchy denoted by AKNS $(-n)$ for $n=0,1,2, \ldots$ with one time $t$ and two space variables $x$ and $y$. In [37], Bogoyavlenski gave a type of AKNS(0) system which can be reduced to a single complex equation that is a compatibility condition for a certain linear system. The reduced equation admits the Lax representation, has breaking solitons, and can be embedded into some $(3+1)$-dimensional complex integrable equation [38]. Strachan also presented a single equation reduced from the same AKNS(0) system as a (2+1)-dimensional generalization of the NLS equation and found one-soliton solution of this system by using Hirota method [39]. All these systems are nonlocal due to the term $D^{-1}$ in the recursion operator. We obtain the Hirota bilinear form of these systems and obtain one- and two-soliton solutions for $n=0,1,2$. We then find all possible local and nonlocal reductions of the negative AKNS hierarchy for $n=0,1,2$. There are in total 30 reduced equations for $n=0,1,2$. All these equations constitute new examples of $(2+1)$-dimensional integrable system of equations. There exists only one type of local reductions where the second dynamical variable is related to the complex conjugation of the other variable. By the use of constraint equations we obtain one-soliton solutions of the local and nonlocal reduced equations from the one-soliton solutions of the negative AKNS system of equations. There are solutions which develop singularities in a finite time and there are also solutions which are finite and bounded depending on the parameters of the one-soliton solutions.

\section{Negative AKNS system}

The AKNS hierarchy [9] can be written as

$$
u_{t_{n}}=\mathcal{R}^{n} u_{x}(n=0,1,2, \ldots), u=\left(\begin{array}{c}
p \\
q
\end{array}\right) \text { i.e. }\left(\begin{array}{l}
p_{t_{N}} \\
q_{t_{N}}
\end{array}\right)=\mathcal{R}^{N-1}\left(\begin{array}{l}
p_{x} \\
q_{x}
\end{array}\right),
$$

where $\mathcal{R}$ is the recursion operator,

$$
\mathcal{R}=\left(\begin{array}{cc}
-p D^{-1} q+\frac{1}{2} D & -p D^{-1} p \\
q D^{-1} q & q D^{-1} p-\frac{1}{2} D
\end{array}\right) .
$$

Here $D$ is the total $x$-derivative and $D^{-1}=\int^{x}$ (standard anti-derivative).

Writing (1.2) in the following form

$$
\mathcal{R}\left(u_{t_{n}}\right)-a \mathcal{R}^{n}\left(u_{x}\right)=b u_{y} \text { for } n=0,1,2, \ldots,
$$

where $u=\left(\begin{array}{l}p \\ q\end{array}\right)$, here $a, b$ are any constants, we obtain $(2+1)$-dimensional negative AKNS $(-n)$ systems for $n=0,1,2, \ldots$ In this work we will only consider the systems for $n=0,1,2$.

(1) $(n=0)(2+1)$-AKNS(0) system:

When $n=0$, Eq. (2.1) reduces to $\mathcal{R}\left(u_{t}\right)-a u_{x}=b u_{y}$. This yields the system

$$
\begin{aligned}
& b p_{y}=\frac{1}{2} p_{t x}-a p_{x}-p D^{-1}(p q)_{t}, \\
& b q_{y}=-\frac{1}{2} q_{t x}-a q_{x}+q D^{-1}(p q)_{t} .
\end{aligned}
$$


(2) $(n=1)(2+1)-\mathbf{A K N S}(-\mathbf{1})$ system:

When $n=1$, Eq. (2.1) reduces to $\mathcal{R}\left(u_{t}-a u_{x}\right)=b u_{y}$. Letting $u_{t}-a u_{x}=\omega$, where $\omega=\left(\begin{array}{c}\omega_{1} \\ \omega_{2}\end{array}\right)$ we have

$$
u_{t}-a u_{x}=\omega, \quad \mathcal{R} \omega=b u_{y} .
$$

This yields the system

$$
\begin{aligned}
\omega_{1} & =p_{t}-a p_{x} \\
\omega_{2} & =q_{t}-a q_{x} \\
b p_{y} & =\frac{1}{2} \omega_{1, x}-p D^{-1}\left(q \omega_{1}+p \omega_{2}\right) \\
b q_{y} & =-\frac{1}{2} \omega_{2, x}+q D^{-1}\left(q \omega_{1}+p \omega_{2}\right) .
\end{aligned}
$$

Inserting $\omega_{1}$ and $\omega_{2}$ we obtain the system

$$
\begin{aligned}
& b p_{y}=\frac{1}{2} p_{t x}-\frac{a}{2} p_{x x}+a p^{2} q-p D^{-1}(p q)_{t}, \\
& b q_{y}=-\frac{1}{2} q_{t x}+\frac{a}{2} q_{x x}-a p q^{2}+q D^{-1}(p q)_{t} .
\end{aligned}
$$

(3) $(n=2)(2+1)-\mathbf{A K N S}(-2)$ system:

When $n=2$, Eq. (2.1) reduces to $\mathcal{R}\left(u_{t}-a \mathcal{R} u_{x}\right)=b u_{y}$. Letting $u_{t}-a \mathcal{R} u_{x}=\omega$, where $\omega=\left(\begin{array}{c}\omega_{1} \\ \omega_{2}\end{array}\right)$ we have

$$
u_{t}-a \mathcal{R} u_{x}=\omega, \quad \mathcal{R} \omega=b u_{y} .
$$

This yields the system

$$
\begin{aligned}
\omega_{1} & =p_{t}-a\left(-p^{2} q+\frac{1}{2} p_{x x}\right) \\
\omega_{2} & =q_{t}-a\left(p q^{2}-\frac{1}{2} q_{x x}\right) \\
b p_{y} & =\frac{1}{2} \omega_{1, x}-p D^{-1}\left(q \omega_{1}+p \omega_{2}\right) \\
b q_{y} & =-\frac{1}{2} \omega_{2, x}+q D^{-1}\left(q \omega_{1}+p \omega_{2}\right) .
\end{aligned}
$$

Inserting $\omega_{1}$ and $\omega_{2}$ we obtain the system

$$
\begin{aligned}
& b p_{y}=\frac{1}{2} p_{t x}-\frac{a}{4} p_{x x x}+\frac{3 a}{2} p q p_{x}-p D^{-1}(p q)_{t} \\
& b q_{y}=-\frac{1}{2} q_{t x}-\frac{a}{4} q_{x x x}+\frac{3 a}{2} p q q_{x}+q D^{-1}(p q)_{t} .
\end{aligned}
$$

\section{Hirota method for negative AKNS system}

To obtain the Hirota bilinear form for the negative $\operatorname{AKNS}(-n)$ system, with $n=0,1$, and $n=2$, we let

$$
p=\frac{g}{f}, \quad q=\frac{h}{f}
$$

and

$$
\frac{g h}{f^{2}}=-\left(\frac{f_{x}}{f}\right)_{x}+\beta,
$$

where $\beta$ is an arbitrary constant.

(1) $(n=0)$ Hirota bilinear form for $(2+1)$-AKNS(0) system:

Using (3.1) and (3.2) in Eqs. (2.2) and (2.3) we have

$$
\begin{aligned}
& b\left(f g_{y}-g f_{y}\right)=\frac{1}{2}\left(f g_{t x}-g_{t} f_{x}-g_{x} f_{t}+g f_{t x}\right)-a\left(f g_{x}-g f_{x}\right), \\
& b\left(f h_{y}-h f_{y}\right)=-\frac{1}{2}\left(f h_{t x}-h_{t} f_{x}-h_{x} f_{t}+h f_{t x}\right)-a\left(f h_{x}-h f_{x}\right) .
\end{aligned}
$$


Hence we obtain the Hirota bilinear form as

$$
\begin{aligned}
& P_{1}(D)\{g \cdot f\} \equiv\left(b D_{y}-\frac{1}{2} D_{t} D_{x}+a D_{x}\right)\{g \cdot f\}=0, \\
& P_{2}(D)\{h \cdot f\} \equiv\left(b D_{y}+\frac{1}{2} D_{t} D_{x}+a D_{x}\right)\{h \cdot f\}=0, \\
& P_{3}(D)\{f \cdot f\} \equiv\left(D_{x}^{2}-2 \beta\right)\{f \cdot f\}=-2 g h .
\end{aligned}
$$

(2) $(n=1)$ Hirota bilinear form for $(2+1)$-AKNS(-1) system:

Using (3.1) and (3.2) in Eqs. (2.5) and (2.6) we get

$$
\begin{aligned}
& b\left(f g_{y}-g f_{y}\right)=\frac{1}{2}\left(f g_{t x}-g_{t} f_{x}-g_{x} f_{t}+g f_{t x}\right)-\frac{a}{2}\left(f g_{x x}-2 f_{x} g_{x}+g f_{x x}-2 \beta g f\right), \\
& b\left(f h_{y}-h f_{y}\right)=-\frac{1}{2}\left(f h_{t x}-h_{t} f_{x}-h_{x} f_{t}+h f_{t x}\right)+\frac{a}{2}\left(f h_{x x}-2 f_{x} h_{x}+h f_{x x}-2 \beta h f\right) .
\end{aligned}
$$

Hence we obtain the Hirota bilinear form as

$$
\begin{aligned}
& P_{1}(D)\{g \cdot f\} \equiv\left(b D_{y}-\frac{1}{2} D_{t} D_{x}+\frac{a}{2} D_{x}^{2}-a \beta\right)\{g \cdot f\}=0, \\
& P_{2}(D)\{h \cdot f\} \equiv\left(b D_{y}+\frac{1}{2} D_{t} D_{x}-\frac{a}{2} D_{x}^{2}+a \beta\right)\{h \cdot f\}=0, \\
& P_{3}(D)\{f \cdot f\} \equiv\left(D_{x}^{2}-2 \beta\right)\{f \cdot f\}=-2 g h .
\end{aligned}
$$

(3) $(n=2)$ Hirota bilinear form for $(2+1)$-AKNS(-2) system:

Using (3.1) and (3.2) in Eqs. (2.8) and (2.9) we have

$$
\begin{aligned}
& 4 b\left(f g_{y}-g f_{y}\right)=2\left(f g_{t x}-g_{t} f_{x}-g_{x} f_{t}+g f_{t x}\right)-a\left(f g_{x x x}+3 f_{x x} g_{x}-3 g_{x x} f_{x}-g f_{x x x}\right)+6 a \beta\left(g_{x} f-g f_{x}\right), \\
& 4 b\left(f h_{y}-h f_{y}\right)=-2\left(f h_{t x}-h_{t} f_{x}-h_{x} f_{t}+h f_{t x}\right)-a\left(f h_{x x x}-3 f_{x} h_{x x}+3 h_{x} f_{x x}-h f_{x x x}\right)+6 a \beta\left(h_{x} f-h f_{x}\right) .
\end{aligned}
$$

Hence we obtain the Hirota bilinear form as

$$
\begin{aligned}
& P_{1}(D)\{g \cdot f\} \equiv\left(b D_{y}-\frac{1}{2} D_{t} D_{x}+\frac{a}{4} D_{x}^{3}-\frac{3 a}{2} \beta D_{x}\right)\{g \cdot f\}=0, \\
& P_{2}(D)\{g \cdot f\} \equiv\left(b D_{y}+\frac{1}{2} D_{t} D_{x}+\frac{a}{4} D_{x}^{3}-\frac{3 a}{2} \beta D_{x}\right)\{h \cdot f\}=0, \\
& P_{3}(D)\{f \cdot f\} \equiv\left(D_{x}^{2}-2 \beta\right)\{f \cdot f\}=-2 g h .
\end{aligned}
$$

After having Hirota bilinear forms (3.5)-(3.7), (3.10)-(3.12), and (3.15)-(3.17), next step is to find the functions $g$, $h$, and $f$ by using the Hirota method (see Sec. VI).

\section{Local reductions}

It is straightforward to show that there exist no consistent local reductions in the form of $q(x, y, t)=\sigma p(x, y, t)$ for all $n=0,1,2$. Here we will give the local reductions in the form of $q(x, y, t)=\sigma \bar{p}(x, y, t)$ for all $n=0,1,2$ where $\sigma$ is any real constant.

(1) Local reductions for the system $n=0$ :

Let $q(x, y, t)=\sigma \bar{p}(x, y, t)$ then two coupled Eqs. (2.2) and (2.3) reduce consistently to the following single equation:

$$
b p_{y}=\frac{1}{2} p_{t x}-a p_{x}-\sigma p D^{-1}(p \bar{p})_{t}
$$

where $\sigma$ is any real constant and a bar over a letter denotes complex conjugation. Here $a$ and $b$ are pure imaginary numbers. In [37], Bogoyavlenski presented the system

$$
i u_{t}-u_{x y}-2 u \partial_{x}^{-1}(u v)_{y}=0
$$




$$
i v_{t}+v_{x y}+2 v \partial_{x}^{-1}(u v)_{y}=0 .
$$

Note that if we interchange the variables $t$ and $y$, take $a=0$ and $b=\frac{i}{2}$ in the system (2.2) and (2.3) we exactly get this system. Bogoyavlenski also mentioned about the reduction $u=\alpha \bar{v}, \alpha \in \mathbb{R}$ and obtained the single equation

$$
v_{t}=i v_{x y}+2 i \alpha v \partial_{x}^{-1}|v|_{y}^{2} \text {. }
$$

This equation has breaking solitons and Lax representation.

(2) Local reductions for the system $n=1$ :

Let $q(x, y, t)=\sigma \bar{p}(x, y, t)$ then two coupled Eqs. (2.5) and (2.6) reduce consistently to the following single equation:

$$
b p_{y}=\frac{1}{2} p_{t x}-\frac{a}{2} p_{x x}+a \sigma p^{2} \bar{p}-\sigma p D^{-1}(p \bar{p})_{t},
$$

where $\sigma$ is any real constant and a bar over a letter denotes complex conjugation. Here $a$ is a real and $b$ is a pure imaginary number.

(3) Local reductions for the system $n=2$ :

Let $q(x, y, t)=\sigma \bar{p}(x, y, t)$ then two coupled Eqs. (2.8) and (2.9) reduce consistently to the following single equation:

$$
b p_{y}=\frac{1}{2} p_{t x}-\frac{a}{4} p_{x x x}+\frac{3 a}{2} \sigma p \bar{p} p_{x}-\sigma p D^{-1}(p \bar{p})_{t},
$$

where $\sigma$ is any real constant and a bar over a letter denotes complex conjugation. Here $a$ and $b$ are pure imaginary numbers.

\section{Nonlocal reductions}

In order to have consistent nonlocal reductions we use the following representation for $D^{-1}$

$$
D^{-1} F=\frac{1}{2}\left(\int_{-\infty}^{x}-\int_{x}^{\infty}\right) F\left(x^{\prime}, y, t\right) d x^{\prime} .
$$

We define the quantity $\rho(x, y, t)$ which is invariant under the discrete transformations $x \rightarrow \epsilon_{1} x, y \rightarrow \epsilon_{2} y$, and $t \rightarrow \epsilon_{3} t$ as

$$
\rho(x, y, t)=D^{-1} p p^{\epsilon} \equiv\left(\int_{-\infty}^{x}-\int_{x}^{\infty}\right) p\left(x^{\prime}, y, t\right) p\left(\epsilon_{1} x^{\prime}, \epsilon_{2} y, \epsilon_{3} t\right) d x^{\prime},
$$

where $\epsilon_{1}^{2}=\epsilon_{2}^{2}=\epsilon_{3}^{2}=1$. It is easy to show that

$$
\rho\left(\epsilon_{1} x, \epsilon_{2} y, \epsilon_{3} t\right)=\epsilon_{1} \rho(x, y, t) .
$$

(1) Nonlocal reductions for the system $n=0$ :

(a) Let $q(x, y, t)=\sigma p\left(\epsilon_{1} x, \epsilon_{2} y, \epsilon_{3} t\right)$ then two coupled Eqs. (2.2) and (2.3) reduce consistently to the following single equation:

$$
b p_{y}=\frac{1}{2} p_{t x}-a p_{x}-\sigma p D^{-1}\left(p p^{\epsilon}\right)_{t}
$$

where $\sigma$ is any real constant and $p^{\epsilon}=p\left(\epsilon_{1} x, \epsilon_{2} y, \epsilon_{3} t\right)$. The above reduced equation is valid only when $\epsilon_{3}=-1$ and $\epsilon_{1} \epsilon_{2}=1$. We have only two possible cases; $p^{\epsilon}=p(x, y,-t)$ and $p^{\epsilon}=p(-x,-y,-t)$ for time reversal and time and space reversals respectively.

(b) Let $q(x, y, t)=\sigma \bar{p}\left(\epsilon_{1} x, \epsilon_{2} y, \epsilon_{3} t\right)$ then two coupled Eqs. (2.2) and (2.3) reduce consistently to the following single equation:

$$
b p_{y}=\frac{1}{2} p_{t x}-a p_{x}-\sigma p D^{-1}\left(p \bar{p}^{\epsilon}\right)_{t}
$$

where $\sigma$ is any real constant. This reduction is valid only when

$$
\epsilon_{1} \epsilon_{2} \epsilon_{3} \bar{b}=-b, \quad \epsilon_{3} \bar{a}=-a .
$$

In this case we have seven different time and space reversals:

(i) $p^{\epsilon}(x, y, t)=p(-x, y, t)$, where $a$ is pure imaginary and $b$ is real.

(ii) $p^{\epsilon}(x, y, t)=p(x,-y, t)$, where $a$ is pure imaginary and $b$ is real.

(iii. $p^{\epsilon}(x, y, t)=p(x, y,-t)$, where $a$ and $b$ are real.

(iv) $p^{\epsilon}(x, y, t)=p(-x,-y, t)$, where $a$ and $b$ are pure imaginary.

(v) $p^{\epsilon}(x, y, t)=p(-x, y,-t)$, where $a$ is real and $b$ is pure imaginary.

(vi) $p^{\epsilon}(x, y, t)=p(x,-y,-t)$, where $a$ is real and $b$ is pure imaginary.

(vii) $p^{\epsilon}(x, y, t)=p(-x,-y,-t)$, where $a$ and $b$ are real. 
Each case above gives a nonlocal equation in the form of (5.5) in $2+1$ dimensions.

In [40] nonlocal reduction given in (v) and corresponding nonlocal equation have been considered for $\beta=-\frac{1}{2}$ in (3.7). Soliton solution have been also found.

(2) Nonlocal reductions for the system $n=1$ :

(a) Let $q(x, y, t)=\sigma p\left(\epsilon_{1} x, \epsilon_{2} y, \epsilon_{3} t\right)$ then two coupled Eqs. (2.5) and (2.6) reduce consistently to the following single equation:

$$
b p_{y}=\frac{1}{2} p_{t x}-\frac{a}{2} p_{x x}+a \sigma p^{2} p^{\epsilon}-\sigma p D^{-1}\left(p p^{\epsilon}\right)_{t},
$$

where $\sigma$ is any real constant and $p^{\epsilon}=p\left(\epsilon_{1} x, \epsilon_{2} y, \epsilon_{3} t\right)$. The above reduced equation is valid only when $\epsilon_{2}=-1$ and $\epsilon_{1} \epsilon_{3}=1$. We have only two possible cases; $p^{\epsilon}=p(x,-y, t)$ and $p^{\epsilon}=p(-x,-y,-t)$ for space reversal and time and space reversals respectively.

(b) Let $q(x, y, t)=\sigma \bar{p}\left(\epsilon_{1} x, \epsilon_{2} y, \epsilon_{3} t\right)$ then two coupled Eqs. (2.5) and (2.6) reduce consistently to the following single equation:

$$
b p_{y}=\frac{1}{2} p_{t x}-\frac{a}{2} p_{x x}+a \sigma p^{2} \bar{p}^{\epsilon}-\sigma p D^{-1}\left(p \bar{p}^{\epsilon}\right)_{t},
$$

where $\sigma$ is any real constant. This reduction is valid only when

$$
\epsilon_{1} \epsilon_{2} \epsilon_{3} \bar{b}=-b, \quad \epsilon_{1} \epsilon_{3} \bar{a}=a .
$$

In this case we have seven different time and space reversals:

(i) $p^{\epsilon}(x, y, t)=p(-x, y, t)$, where $a$ is pure imaginary and $b$ is real.

(ii) $p^{\epsilon}(x, y, t)=p(x,-y, t)$, where $a$ and $b$ are real.

(iii) $p^{\epsilon}(x, y, t)=p(x, y,-t)$, where $a$ is pure imaginary and $b$ are real.

(iv) $p^{\epsilon}(x, y, t)=p(-x,-y, t)$, where $a$ and $b$ are pure imaginary.

(v) $p^{\epsilon}(x, y, t)=p(-x, y,-t)$, where $a$ is real and $b$ is pure imaginary.

(vi) $p^{\epsilon}(x, y, t)=p(x,-y,-t)$, where $a$ and $b$ are pure imaginary.

(vii) $p^{\epsilon}(x, y, t)=p(-x,-y,-t)$, where $a$ and $b$ are real.

Each case above gives a nonlocal equation in the form of (5.8) in $2+1$ dimensions.

(3) Nonlocal reductions for the system $n=2$ :

(a) Let $q(x, y, t)=\sigma p\left(\epsilon_{1} x, \epsilon_{2} y, \epsilon_{3} t\right)$ then two coupled Eqs. (2.8) and (2.9) reduce consistently to the following single equation:

$$
b p_{y}=\frac{1}{2} p_{t x}-\frac{a}{4} p_{x x x}+\frac{3 a}{2} \sigma p p^{\epsilon} p_{x}-\sigma p D^{-1}\left(p p^{\epsilon}\right)_{t}
$$

where $\sigma$ is any real constant and $p^{\epsilon}=p\left(\epsilon_{1} x, \epsilon_{2} y, \epsilon_{3} t\right)$. The above reduced equation is valid only when $\epsilon_{3}=-1$ and $\epsilon_{1} \epsilon_{2}=1$. We have only two possible cases; $p^{\epsilon}=p(x, y,-t)$ and $p^{\epsilon}=p(-x,-y,-t)$ for time reversal and time and space reversals respectively.

(b) Let $q(x, y, t)=\sigma \bar{p}\left(\epsilon_{1} x, \epsilon_{2} y, \epsilon_{3} t\right)$ then two coupled Eqs. (2.5) and (2.6) reduce consistently to the following single equation:

$$
b p_{y}=\frac{1}{2} p_{t x}-\frac{a}{4} p_{x x x}+\frac{3 a}{2} \sigma p \bar{p}^{\epsilon} p_{x}-\sigma p D^{-1}\left(p \bar{p}^{\epsilon}\right)_{t},
$$

where $\sigma$ is any real constant. This reduction is valid only when

$$
\epsilon_{1} \epsilon_{2} \epsilon_{3} \bar{b}=-b, \quad \epsilon_{3} \bar{a}=-a .
$$

In this case we have seven different time and space reversals:

(i) $p^{\epsilon}(x, y, t)=p(-x, y, t)$, where $a$ is pure imaginary and $b$ is real.

(ii) $p^{\epsilon}(x, y, t)=p(x,-y, t)$, where $a$ is pure imaginary and $b$ is real.

(iii) $p^{\epsilon}(x, y, t)=p(x, y,-t)$, where $a$ and $b$ are real.

(iv) $p^{\epsilon}(x, y, t)=p(-x,-y, t)$, where $a$ and $b$ are pure imaginary.

(v) $p^{\epsilon}(x, y, t)=p(-x, y,-t)$, where $a$ is real and $b$ is pure imaginary.

(vi) $p^{\epsilon}(x, y, t)=p(x,-y,-t)$, where $a$ is real and $b$ is pure imaginary.

(vii) $p^{\epsilon}(x, y, t)=p(-x,-y,-t)$, where $a$ and $b$ are real.

Each case above gives a nonlocal equation in the form of (5.11) in $2+1$ dimensions. At the end we obtain 27 nonlocal equations from negative AKNS hierarchy in $2+1$ dimensions.

Remark 1. In all the above nonlocal equations we can use $D^{-1}=\int^{x}$ when there exist only $y$ and $t$ reversals, $p^{\epsilon}=$ $p\left(x, \epsilon_{2} y, \epsilon_{3} t\right)$.

\section{Soliton solutions for negative AKNS hierarchy}

In the following sections we solve the Hirota bilinear equations of $(2+1)$-AKNS $(-n)$ systems for $n=0,1,2$ when $\beta=0$ and find one- and two-soliton solutions. 
6.1. One-soliton solution of $(2+1)-\operatorname{AKNS}(-n)$ system $(n=0,1,2)$

Here we will present how to find one-soliton solution of $(2+1)$-AKNS(0) system. For $n=1$ and $n=2$ the steps for finding one-soliton solution are same with $n=0$ case except the dispersion relations. $f$,

Consider the system (3.5)-(3.7). To find one-soliton solution we use the following expansions for the functions $g$, $h$, and

$$
g=\varepsilon g_{1}, \quad h=\varepsilon h_{1}, \quad f=1+\varepsilon^{2} f_{2},
$$

where

$$
g_{1}=e^{\theta_{1}}, \quad h_{1}=e^{\theta_{2}}, \quad \theta_{i}=k_{i} x+\tau_{i} y+\omega_{i} t+\delta_{i}, i=1,2 .
$$

When we substitute (6.1) into the Eqs. (3.5)-(3.7), we obtain the coefficients of $\varepsilon$ as

$$
\begin{aligned}
& P_{1}(D)\left\{g_{1} \cdot 1\right\}=b g_{1, y}-\frac{1}{2} g_{1, x t}+a g_{1, x}=0, \\
& P_{2}(D)\left\{h_{1} \cdot 1\right\}=b h_{1, y}+\frac{1}{2} h_{1, x t}+a h_{1, x}=0,
\end{aligned}
$$

yielding the dispersion relations

$$
\tau_{1}=\frac{1}{b}\left(\frac{1}{2} k_{1} \omega_{1}-a k_{1}\right), \quad \tau_{2}=\frac{1}{b}\left(-\frac{1}{2} k_{2} \omega_{2}-a k_{2}\right) .
$$

From the coefficient of $\varepsilon^{2}$

$$
f_{2, x x}=-g_{1} h_{1}
$$

we obtain the function $f_{2}$ as

$$
f_{2}=-\frac{e^{\left(k_{1}+k_{2}\right) x+\left(\tau_{1}+\tau_{2}\right) y+\left(\omega_{1}+\omega_{2}\right) t+\delta_{1}+\delta_{2}}}{\left(k_{1}+k_{2}\right)^{2}} .
$$

The coefficients of $\varepsilon^{3}$ vanish with the dispersion relations and (6.7). From the coefficient of $\varepsilon^{4}$ we get

$$
f_{2} f_{2, x x}-f_{2, x}^{2}=0
$$

and this equation also vanishes immediately due to the dispersion relations and (6.7). Without loss of generality let us also take $\varepsilon=1$. Hence a pair of solutions of $(2+1)-\operatorname{AKNS}(0)$ system $(2.2)$ and (2.3) is given by $(p(x, y, t), q(x, y, t))$ where

$$
p(x, y, t)=\frac{e^{\theta_{1}}}{1+A e^{\theta_{1}+\theta_{2}}}, \quad q(x, y, t)=\frac{e^{\theta_{2}}}{1+A e^{\theta_{1}+\theta_{2}}},
$$

with $\theta_{i}=k_{i} x+\tau_{i} y+\omega_{i} t+\delta_{i}, i=1,2, \tau_{1}=\frac{1}{b}\left(\frac{1}{2} k_{1} \omega_{1}-a k_{1}\right), \tau_{2}=\frac{1}{b}\left(-\frac{1}{2} k_{2} \omega_{2}-a k_{2}\right)$, and $A=-\frac{1}{\left(k_{1}+k_{2}\right)^{2}}$. Here $k_{i}, \omega_{i}$, and $\delta_{i}$, $i=1,2$ are arbitrary complex numbers.

For $n=1$ that is for the system (2.5) and (2.6) one-soliton solution is given by (6.9) where $\theta_{i}=k_{i} x+\tau_{i} y+\omega_{i} t+\delta_{i}, i=1,2$ with

$$
\tau_{1}=\frac{1}{b}\left(\frac{1}{2} k_{1} \omega_{1}-\frac{a}{2} k_{1}^{2}\right), \quad \tau_{2}=\frac{1}{b}\left(-\frac{1}{2} k_{2} \omega_{2}+\frac{a}{2} k_{2}^{2}\right) .
$$

For $n=2$ that is for the system (2.8) and (2.9) one-soliton solution is again given by (6.9) where $\theta_{i}=k_{i} x+\tau_{i} y+\omega_{i} t+\delta_{i}$, $i=1,2$ with

$$
\tau_{1}=\frac{1}{b}\left(\frac{1}{2} k_{1} \omega_{1}-\frac{a}{4} k_{1}^{3}\right), \quad \tau_{2}=\frac{1}{b}\left(-\frac{1}{2} k_{2} \omega_{2}-\frac{a}{4} k_{2}^{3}\right) .
$$

6.2. Two-soliton solution of $(2+1)-\operatorname{AKNS}(-n)$ system $(n=0,1,2)$

Here as in the previous section, we will only deal with $(2+1)$-AKNS $(0)$ system and find two-soliton solution of this system. For $n=1$ and $n=2$ we have the same form of two-soliton solution only with difference of the dispersion relations.

Consider the system (3.5)-(3.7). For two-soliton solution, we take

$$
g=\varepsilon g_{1}+\varepsilon^{3} g_{3}, \quad h=\varepsilon h_{1}+\varepsilon^{3} h_{3}, \quad f=1+\varepsilon^{2} f_{2}+\varepsilon^{4} f_{4},
$$

where

$$
g_{1}=e^{\theta_{1}}+e^{\theta_{2}}, \quad h_{1}=e^{\eta_{1}}+e^{\eta_{2}},
$$

with $\theta_{i}=k_{i} x+\tau_{i} y+\omega_{i} t+\delta_{i}, \eta_{i}=\ell_{i} x+s_{i} y+m_{i} t+\alpha_{i}$ for $i=1,2$. When we insert above expansions into (3.5)-(3.7), we get the coefficients of $\varepsilon^{n}, 1 \leq n \leq 8$ as

$$
\varepsilon: b g_{1, y}-\frac{1}{2} g_{1, x t}+a g_{1, x}=0
$$




$$
\begin{aligned}
& b h_{1, y}+\frac{1}{2} h_{1, x t}+a h_{1, x}=0 \\
& \varepsilon^{2}: f_{2, x x}+g_{1} h_{1}=0 \\
& \varepsilon^{3}: b\left(g_{1, y} f_{2}-g_{1} f_{2, y}\right)-\frac{1}{2}\left(g_{1, x t} f_{2}-g_{1, t} f_{2, x}-g_{1, x} f_{2, t}+g_{1} f_{2, x t}\right)+a\left(g_{1, x} f_{2}-g_{1} f_{2, x}\right)+b g_{3, y}-\frac{1}{2} g_{3, x t}+a g_{3, x}=0, \\
& b\left(h_{1, y} f_{2}-h_{1} f_{2, y}\right)+\frac{1}{2}\left(h_{1, x t} f_{2}-h_{1, t} f_{2, x}-h_{1, x} f_{2, t}+h_{1} f_{2, x t}\right)+a\left(h_{1, x} f_{2}-h_{1} f_{2, x}\right)+b h_{3, y}+\frac{1}{2} h_{3, x t}+a h_{3, x}=0, \\
& \varepsilon^{4}: f_{2} f_{2, x x}-f_{2, x}^{2}+f_{4, x x}+g_{1} h_{3}+g_{3} h_{1}=0, \\
& \varepsilon^{5}: b\left(g_{1, y} f_{4}-g_{1} f_{4, y}\right)-\frac{1}{2}\left(g_{1, x t} f_{4}-g_{1, t} f_{4, x}-g_{1, x} f_{4, t}+g_{1} f_{4, x t}\right)+a\left(g_{1, x} f_{4}-g_{1} f_{4, x}\right) \\
& +b\left(g_{3, y} f_{2}-g_{3} f_{2, y}\right)-\frac{1}{2}\left(g_{3, x t} f_{2}-g_{3, t} f_{2, x}-g_{3, x} f_{2, t}+g_{3} f_{2, x t}\right)+a\left(g_{3, x} f_{2}-g_{3} f_{2, x}\right)=0, \\
& b\left(h_{1, y} f_{4}-h_{1} f_{4, y}\right)+\frac{1}{2}\left(h_{1, x t} f_{4}-h_{1, t} f_{4, x}-h_{1, x} f_{4, t}+h_{1} f_{4, x t}\right)+a\left(h_{1, x} f_{4}-h_{1} f_{4, x}\right) \\
& +b\left(h_{3, y} f_{2}-h_{3} f_{2, y}\right)+\frac{1}{2}\left(h_{3, x t} f_{2}-h_{3, t} f_{2, x}-h_{3, x} f_{2, t}+h_{3} f_{2, x t}\right)+a\left(h_{3, x} f_{2}-h_{3} f_{2, x}\right)=0, \\
& \varepsilon^{6}: f_{2, x x} f_{4}-2 f_{2, x} f_{4, x}+f_{2} f_{4, x x}+g_{3} h_{3}=0, \\
& \varepsilon^{7}: b\left(g_{3, y} f_{4}-g_{3} f_{4, y}\right)-\frac{1}{2}\left(g_{3, x t} f_{4}-g_{3, t} f_{4, x}-g_{3, x} f_{4, t}+g_{3} f_{4, x t}\right)+a\left(g_{3, x} f_{4}-g_{3} f_{4, x}\right)=0, \\
& b\left(h_{3, y} f_{4}-h_{3} f_{4, y}\right)+\frac{1}{2}\left(h_{3, x t} f_{4}-h_{3, t} f_{4, x}-h_{3, x} f_{4, t}+h_{3} f_{4, x t}\right)+a\left(h_{3, x} f_{4}-h_{3} f_{4, x}\right)=0 . \\
& \varepsilon^{8}: f_{4} f_{4, x x}-f_{4, x}^{2}=0 .
\end{aligned}
$$

The Eqs. (6.14) and (6.15) give the dispersion relations

$$
\tau_{i}=\frac{1}{b}\left(\frac{1}{2} k_{i} \omega_{i}-a k_{i}\right), \quad s_{i}=\frac{1}{b}\left(-\frac{1}{2} \ell_{i} m_{i}-a \ell_{i}\right), \quad i=1,2 .
$$

From the coefficient of $\varepsilon^{2}$ we obtain the function $f_{2}$,

$$
f_{2}=e^{\theta_{1}+\eta_{1}+\alpha_{11}}+e^{\theta_{1}+\eta_{2}+\alpha_{12}}+e^{\theta_{2}+\eta_{1}+\alpha_{21}}+e^{\theta_{2}+\eta_{2}+\alpha_{22}}=\sum_{1 \leq i, j \leq 2} e^{\theta_{i}+\eta_{j}+\alpha_{i j}},
$$

where

$$
e^{\alpha_{i j}}=-\frac{1}{\left(k_{i}+\ell_{j}\right)^{2}}, 1 \leq i, j \leq 2 .
$$

The Eqs. (6.17) and (6.18) give the functions $g_{3}$ and $h_{3}$,

$$
g_{3}=A_{1} e^{\theta_{1}+\theta_{2}+\eta_{1}}+A_{2} e^{\theta_{1}+\theta_{2}+\eta_{2}}, \quad h_{3}=B_{1} e^{\theta_{1}+\eta_{1}+\eta_{2}}+B_{2} e^{\theta_{2}+\eta_{1}+\eta_{2}},
$$

where

$$
A_{i}=-\frac{\left(k_{1}-k_{2}\right)^{2}}{\left(k_{1}+\ell_{i}\right)^{2}\left(k_{2}+\ell_{i}\right)^{2}}, \quad B_{i}=-\frac{\left(\ell_{1}-\ell_{2}\right)^{2}}{\left(\ell_{1}+k_{i}\right)^{2}\left(\ell_{2}+k_{i}\right)^{2}}, i=1,2 .
$$

The Eq. (6.19) yields the function $f_{4}$ as

$$
f_{4}=M e^{\theta_{1}+\theta_{2}+\eta_{1}+\eta_{2}},
$$


where

$$
M=\frac{\left(k_{1}-k_{2}\right)^{2}\left(l_{1}-l_{2}\right)^{2}}{\left(k_{1}+l_{1}\right)^{2}\left(k_{1}+l_{2}\right)^{2}\left(k_{2}+l_{1}\right)^{2}\left(k_{2}+l_{2}\right)^{2}} .
$$

Other Eqs. (6.20)-(6.25) vanish immediately by the dispersion relations (6.26) and the functions $f_{2}, f_{4}, g_{3}$, and $h_{3}$.

Take $\varepsilon=1$. Then two-soliton solution of the system (2.2) and (2.3) is given with the pair $(p(x, y, t), q(x, y, t))$,

$$
\begin{aligned}
& p(x, y, t)=\frac{e^{\theta_{1}}+e^{\theta_{2}}+A_{1} e^{\theta_{1}+\theta_{2}+\eta_{1}}+A_{2} e^{\theta_{1}+\theta_{2}+\eta_{2}}}{1+e^{\theta_{1}+\eta_{1}+\alpha_{11}}+e^{\theta_{1}+\eta_{2}+\alpha_{12}}+e^{\theta_{2}+\eta_{1}+\alpha_{21}}+e^{\theta_{2}+\eta_{2}+\alpha_{22}}+M e^{\theta_{1}+\theta_{2}+\eta_{1}+\eta_{2}}}, \\
& q(x, y, t)=\frac{e^{\eta_{1}}+e^{\eta_{2}}+B_{1} e^{\theta_{1}+\eta_{1}+\eta_{2}}+B_{2} e^{\theta_{2}+\eta_{1}+\eta_{2}}}{1+e^{\theta_{1}+\eta_{1}+\alpha_{11}}+e^{\theta_{1}+\eta_{2}+\alpha_{12}}+e^{\theta_{2}+\eta_{1}+\alpha_{21}}+e^{\theta_{2}+\eta_{2}+\alpha_{22}}+M e^{\theta_{1}+\theta_{2}+\eta_{1}+\eta_{2}}},
\end{aligned}
$$

with $\theta_{i}=k_{i} x+\tau_{i} y+\omega_{i} t+\delta_{i}, \quad \eta_{i}=\ell_{i} x+s_{i} y+m_{i} t+\alpha_{i}$ for $i=1,2$ with the dispersion relations $\tau_{i}=\frac{1}{b}\left(\frac{1}{2} k_{i} \omega_{i}-a k_{i}\right), s_{i}=$ $\frac{1}{b}\left(-\frac{1}{2} \ell_{i} m_{i}-a \ell_{i}\right), i=1,2$. Here $k_{i}, \ell_{i}, \omega_{i}, m_{i}, \delta_{i}$, and $\alpha_{i}, i=1,2$ are arbitrary complex numbers.

For $n=1$ i.e. for the system (2.5) and (2.6) two-soliton solution is given by (6.33) and (6.34) where $\theta_{i}=k_{i} x+\tau_{i} y+\omega_{i} t+$ $\delta_{i}, \eta_{i}=\ell_{i} x+s_{i} y+m_{i} t+\alpha_{i}$ for $i=1,2$ with the dispersion relations

$$
\tau_{i}=\frac{1}{b}\left(\frac{1}{2} k_{i} \omega_{i}-\frac{a}{2} k_{i}^{2}\right), \quad s_{i}=\frac{1}{b}\left(-\frac{1}{2} \ell_{i} m_{i}+\frac{a}{2} \ell_{i}^{2}\right), i=1,2 .
$$

For $n=2$ that is for the system (2.8) and (2.9) two-soliton solution is also given by (6.33) and (6.34) where $\theta_{i}=k_{i} x+\tau_{i} y+$ $\omega_{i} t+\delta_{i}, \eta_{i}=\ell_{i} x+s_{i} y+m_{i} t+\alpha_{i}$ for $i=1,2$ with the dispersion relations

$$
\tau_{i}=\frac{1}{b}\left(\frac{1}{2} k_{i} \omega_{i}-\frac{a}{4} k_{i}^{3}\right), \quad s_{i}=\frac{1}{b}\left(-\frac{1}{2} \ell_{i} m_{i}-\frac{a}{4} \ell_{i}^{3}\right), i=1,2 .
$$

\section{Soliton solutions of reduced equations}

In our studies of nonlocal NLS and nonlocal mKdV equations we introduced a general method [32-36] to obtain soliton solutions of nonlocal integrable equation. This method consists of three main steps:

- Find a consistent reduction formula which reduces the integrable system of equations to integrable nonlocal equations.

- Find soliton solutions of the system of equations by use of the Hirota bilinear method or by inverse scattering transform technique, or by use of Darboux transformation.

- Use the reduction formulas on the soliton solutions of the system of equations to obtain the soliton solutions of the reduced nonlocal equations. By this way one obtains many different relations among the soliton parameters of the system of equations.

In the following sections we mainly follow the above method in obtaining the soliton solutions of AKNS $(-n)$ systems for $n=0,1$, and $n=2$ by using Type 1 and Type 2 approaches given in [33].

\subsection{One-soliton solutions of local reduced equations}

The constraints that one-soliton solutions of the local Eqs. (4.1), (4.5), and (4.6) which are reduced from AKNS( $-n)$ for $n=0,1$, and $n=2$ systems respectively can be found by the local reduction formula $q(x, y, t)=\sigma \bar{p}(x, y, t)$ that is

$$
\frac{e^{k_{2} x+\tau_{2} y+\omega_{2} t+\delta_{2}}}{1+A e^{\left(k_{1}+k_{2}\right) x+\left(\tau_{1}+\tau_{2}\right) y+\left(\omega_{1}+\omega_{2}\right) t+\delta_{1}+\delta_{2}}}=\frac{\sigma e^{\bar{k}_{1} x+\bar{\tau}_{1} y+\bar{\omega}_{1} t+\bar{\delta}_{1}}}{1+\bar{A} e^{\left(\bar{k}_{1}+\bar{k}_{2}\right) x+\left(\bar{\tau}_{1}+\bar{\tau}_{2}\right) y+\left(\bar{\omega}_{1}+\bar{\omega}_{2}\right) t+\bar{\delta}_{1}+\bar{\delta}_{2}}} .
$$

If we use the Type 1 approach, we obtain the following constraints:

$$
\text { 1) } \left.k_{2}=\bar{k}_{1}, \quad \text { 2) } \omega_{2}=\bar{\omega}_{1}, \quad 3\right) e^{\delta_{2}}=\sigma e^{\bar{\delta}_{1}},
$$

so that the equality (7.1) is satisfied for each $n=0,1$, and $n=2$. Note that under the above constraints, the dispersion relations give $\tau_{2}=\bar{\tau}_{1}$. Hence one-soliton solutions of (4.1), (4.5), and (4.6) are given by

$$
p(x, y, t)=\frac{e^{k_{1} x+\tau_{1} y+\omega_{1} t+\delta_{1}}}{1-\frac{\sigma}{\left(k_{1}+\bar{k}_{1}\right)^{2}} e^{\left(k_{1}+\bar{k}_{1}\right) x+\left(\tau_{1}+\bar{\tau}_{1}\right) y+\left(\omega_{1}+\bar{\omega}_{1}\right) t+\delta_{1}+\bar{\delta}_{1}}},
$$

where

i. for $n=0, a$ and $b$ are pure imaginary numbers and $\tau_{1}=\frac{1}{b}\left(\frac{1}{2} k_{1} \omega_{1}-a k_{1}\right)$,

ii. for $n=1, a$ is a real and $b$ is a pure imaginary number and $\tau_{1}=\frac{1}{b}\left(\frac{1}{2} k_{1} \omega_{1}-\frac{a}{2} k_{1}^{2}\right)$,

iii. for $n=2, a$ and $b$ are pure imaginary numbers and $\tau_{1}=\frac{1}{b}\left(\frac{1}{2} k_{1} \omega_{1}-\frac{a}{4} k_{1}^{3}\right)$. 
If $\operatorname{sign}(\sigma)<0$ we can let

$$
\sigma=-\left(k_{1}+\bar{k}_{1}\right)^{2} e^{\mu},
$$

where $\mu$ is another real constant. Then the above one-soliton solution becomes

$$
p(x, y, t)=\frac{e^{\phi}}{2 \cosh \theta},
$$

where

$$
\begin{aligned}
& \theta=\frac{1}{2}\left[\left(k_{1}+\bar{k}_{1}\right) x+\left(\tau_{1}+\bar{\tau}_{1}\right) y+\left(w_{1}+\bar{w}_{1}\right) t+\delta_{1}+\bar{\delta}_{1}+\mu\right], \\
& \phi=\frac{1}{2}\left[\left(k_{1}-\bar{k}_{1}\right) x+\left(\tau_{1}-\bar{\tau}_{1}\right) y+\left(w_{1}-\bar{w}_{1}\right) t+\delta_{1}-\bar{\delta}_{1}-\mu\right],
\end{aligned}
$$

Hence one-soliton solutions of the locally reduced equations for $n=0,1,2$ are finite and bounded when sign $(\sigma)=-1$. The norm of $p$ becomes

$$
|p(x, y, t)|^{2}=\frac{e^{-\mu}}{4 \cosh ^{2} \theta} .
$$

Note that in [39], Strachan studied one-soliton solutions of the generalization of NLS equation given by

$$
\begin{aligned}
i \partial_{t} \psi & =\partial_{x y} \psi+V(|\psi|) \psi \\
\partial_{x} V & =2 \partial_{y}|\psi|^{2} .
\end{aligned}
$$

Indeed the single Eq. (4.1) is equivalent to the above system if we interchange the variables $t$ and $y$, take $a=0, \sigma=-1$, and $b=\frac{i}{2}$ in (4.1). To obtain one-soliton solution, Strachan applied the Hirota method directly on the Hirota bilinear form of this single equation. One of the solutions given in [39] is same with our one-soliton solution (7.3). Notice that there is a typo in the Hirota bilinear form of the (7.9) and so in the dispersion relation in [39]. In addition to that solution, Strachan obtained more general solution by changing the solution ansatz.

\subsection{One-soliton solutions of nonlocal reduced equations}

Firstly let us consider the nonlocal reduction $q(x, y, t)=\sigma p\left(\epsilon_{1} x, \epsilon_{2} y, \epsilon_{3} t\right)$. Here the constraints that one-soliton solutions of the nonlocal Eqs. (5.4), (5.7), and (5.10) which are reduced from AKNS( $-n)$ for $n=0,1$, and $n=2$ systems respectively can be found by

$$
\frac{e^{k_{2} x+\tau_{2} y+\omega_{2} t+\delta_{2}}}{1+A e^{\left(k_{1}+k_{2}\right) x+\left(\tau_{1}+\tau_{2}\right) y+\left(\omega_{1}+\omega_{2}\right) t+\delta_{1}+\delta_{2}}}=\frac{\sigma e^{\epsilon_{1} k_{1} x+\epsilon_{2} \tau_{1} y+\epsilon_{3} \omega_{1} t+\delta_{1}}}{1+A e^{\epsilon_{1}\left(k_{1}+k_{2}\right) x+\epsilon_{2}\left(\tau_{1}+\tau_{2}\right) y+\epsilon_{3}\left(\omega_{1}+\omega_{2}\right) t+\delta_{1}+\delta_{2}}},
$$

where $A=-\frac{1}{\left(k_{1}+k_{2}\right)^{2}}$ and $\tau_{i}, i=1,2$ can be written in terms of $k_{i}$ and $\omega_{i}$ due to the dispersion relations of each case $n=0,1,2$.

If we use the Type 1 approach, we obtain

$$
\text { 1) } k_{2}=\epsilon_{1} k_{1}, \text { 2) } \omega_{2}=\epsilon_{3} \omega_{1}, \text { 3) } e^{\delta_{2}}=\sigma e^{\delta_{1}} \text {. }
$$

When we use these constraints with the possibilities for $\left(\epsilon_{1}, \epsilon_{2}, \epsilon_{3}\right)$ given in Sects. 7.4, 7.5, and 7.6 on the dispersion relations of the cases $n=0,1,2$, we get $\tau_{2}=\epsilon_{2} \tau_{1}$.

For $n=0$ we have $\left(\epsilon_{1}, \epsilon_{2}, \epsilon_{3}\right)=(1,1,-1)$ and one-soliton solution of the reduced Eq. (5.4) is

$$
p(x, y, t)=\frac{e^{k_{1} x+\tau_{1} y+\omega_{1} t+\delta_{1}}}{1-\frac{\sigma}{4 k_{1}^{2}}, e^{2 k_{1} x+2 \tau_{1} y+2 \delta_{1}}},
$$

where $\tau_{1}=\frac{1}{b}\left(\frac{1}{2} k_{1} \omega_{1}-a k_{1}\right)$. Assume that all the parameters; $k_{1}, \omega_{1}, \delta_{1}, a$, and $b$ so $\tau_{1}$ are real. Let $\sigma=-4 k_{1}^{2} e^{2 \mu}$ then

$$
p(x, y, t)=\frac{e^{\phi}}{1+e^{2 \theta+2 \mu}}
$$

where $\mu$ is a real constant and

$$
\begin{aligned}
& \phi=k_{1} x+\tau_{1} y+\omega_{1} t+\delta_{1}, \\
& \theta=k_{1} x+\tau_{1} y+\delta_{1} .
\end{aligned}
$$

Eq. (7.13) can further be simplified as

$$
p(x, y, t)=\frac{e^{\omega_{1} t-\mu}}{2 \cosh (\theta+\mu)} .
$$


Hence for the defocusing case, $\operatorname{sign}(\sigma)=-1$, one-soliton solution is bounded for all $t \geq 0$ for $\omega_{1} \leq 0$ and finite for all $(x, y$, t).

For $n=1$ we have $\left(\epsilon_{1}, \epsilon_{2}, \epsilon_{3}\right)=(1,-1,1)$ and one-soliton solution of the reduced Eq. (5.7) is

$$
p(x, y, t)=\frac{e^{k_{1} x+\tau_{1} y+\omega_{1} t+\delta_{1}}}{1-\frac{\sigma}{4 k_{1}^{2}} e^{2 k_{1} x+2 \omega_{1} t+2 \delta_{1}}},
$$

where $\tau_{1}=\frac{1}{b}\left(\frac{1}{2} k_{1} \omega_{1}-\frac{a}{2} k_{1}^{2}\right)$. Assume that all the parameters; $k_{1}, \omega_{1}, \delta_{1}, a$, and $b$ so $\tau_{1}$ are real. Let $\sigma=-4 k_{1}^{2} e^{2 \mu}$ then

$$
p(x, y, t)=\frac{e^{\phi}}{1+e^{2 \theta+2 \mu}},
$$

where $\mu$ is a real constant and

$$
\begin{aligned}
& \phi=k_{1} x+\tau_{1} y+\omega_{1} t+\delta_{1}, \\
& \theta=k_{1} x+\omega_{1} t+\delta_{1},
\end{aligned}
$$

which can be simplified as

$$
p(x, y, t)=\frac{e^{\tau_{1} y-\mu}}{2 \cosh (\theta+\mu)} .
$$

Hence for $\operatorname{sign}(\sigma)=-1$, one-soliton solution is finite for all $(x, y, t)$ but not bounded.

For $n=2$ we have $\left(\epsilon_{1}, \epsilon_{2}, \epsilon_{3}\right)=(1,1,-1)$ and one-soliton solution of the reduced Eq. (5.10) is

$$
p(x, y, t)=\frac{e^{k_{1} x+\tau_{1} y+\omega_{1} t+\delta_{1}}}{1-\frac{\sigma}{4 k_{1}^{2}} e^{2 k_{1} x+2 \tau_{1} y+2 \delta_{1}}},
$$

where $\tau_{1}=\frac{1}{b}\left(\frac{1}{2} k_{1} \omega_{1}-\frac{a}{4} k_{1}^{3}\right)$. Hence, similar to $n=0$ case, the solution (7.22) can be simplified to the form (7.16) with only difference in $\tau_{1}$. And that solution is bounded for all $t \geq 0$ for $\omega_{1} \leq 0$ and finite for all $(x, y, t)$ when $\operatorname{sign}(\sigma)=-1$.

Note that other possibility in each of the cases for $\left(\epsilon_{1}, \epsilon_{2}, \epsilon_{3}\right)$ is $(-1,-1,-1)$. Clearly, because of the definition of the constant $A$, if we use Type 1 approach we obtain trivial solution. Hence we use Type 2 on

$$
\frac{e^{\theta_{2}}}{1+A e^{\theta_{1}+\theta_{2}}}=\sigma \frac{e^{\theta_{1}^{-}}}{1+A e^{\theta_{1}^{-}+\theta_{2}^{-}}} .
$$

From the application of the cross multiplication we get

$$
e^{\theta_{2}}+A e^{2 \delta_{2}} e^{\theta_{1}^{-}}=k e^{\theta_{1}^{-}}+A k e^{2 \delta_{1}} e^{\theta_{2}},
$$

where

$$
\theta_{j}=k_{j} x+\tau_{j} y+\omega_{j} t+\delta_{j}, \quad \theta_{1}^{-}=-k_{j} x-\tau_{j} y-\omega_{j} t+\delta_{j}, \quad j=1,2 .
$$

Hence we obtain the conditions

$$
\text { 1) } A \sigma e^{2 \delta_{1}}=1, \quad \text { 2) } A e^{2 \delta_{2}}=\sigma \text {, }
$$

yielding $e^{\delta_{1}}=\xi_{1} i \frac{\left(k_{1}+k_{2}\right)}{\sqrt{\sigma}}$ and $e^{\delta_{2}}=\xi_{2} i \sqrt{\sigma}\left(k_{1}+k_{2}\right)$ for $\xi_{j}= \pm 1, j=1,2$. Therefore one-soliton solutions of the Eqs. (5.4), (5.7), and (5.10) are given by

$$
p(x, y, t)=\frac{i \xi_{1} e^{k_{1} x+\tau_{1} y+\omega_{1} t}\left(k_{1}+k_{2}\right)}{\sqrt{\sigma}\left(1+\xi_{1} \xi_{2} e^{\left(k_{1}+k_{2}\right) x+\left(\tau_{1}+\tau_{2}\right) y+\left(\omega_{1}+\omega_{2}\right) t}\right)}, \quad \xi_{j}= \pm 1, j=1,2,
$$

with corresponding dispersion relations; (6.5) for $n=0$, (6.10) for $n=1$, and (6.11) for $n=2$. We can further simplify the solution (7.26) as

$$
p(x, y, t)=\frac{e^{\phi+\delta_{1}}}{2 \cosh \theta}
$$

where

$$
\begin{aligned}
& \phi=\frac{1}{2}\left[\left(k_{1}-k_{2}\right) x+\left(\tau_{1}-\tau_{2}\right) y+\left(\omega_{1}-\omega_{2}\right) t\right], \\
& \theta=\frac{1}{2}\left[\left(k_{1}+k_{2}\right) x+\left(\tau_{1}+\tau_{2}\right) y+\left(\omega_{1}+\omega_{2}\right) t\right] .
\end{aligned}
$$

The solution (7.27) is finite if $k_{1}+k_{2}, \tau_{1}+\tau_{2}$, and $\omega_{1}+\omega_{2}$ are real. In addition to that it is bounded if $k_{1}-k_{2}=0, \tau_{1}-\tau_{2}=$ 0 , and $\omega_{1}-\omega_{2} \leq 0$ for $t \geq 0$. For $n=0$ and $n=2$ cases, these conditions are satisfied if $k_{1}, \tau_{1}, \omega_{1}$ are real, $k_{1}=k_{2}, \tau_{1}=$ 
$\tau_{2}, \omega_{2}=-\omega_{1}$, and $\omega_{1} \leq 0$ for $t \geq 0$. For $n=1$ case, they are satisfied if $k_{1}, \tau_{1}, \omega_{1}, a$ are real, $k_{1}=k_{2}, \tau_{1}=\tau_{2}, \omega_{2}=2 a k_{1}-\omega_{1}$, and $\omega_{1}-a k_{1} \leq 0$ for $t \geq 0$.

The second nonlocal reduction formula is $q(x, y, t)=\sigma \bar{p}\left(\epsilon_{1} x, \epsilon_{2} y, \epsilon_{3} t\right)$. The constraints that one-soliton solutions of the nonlocal Eqs. (5.5), (5.8), and (5.11) which are reduced from $\operatorname{AKNS}(-n)$ for $n=0,1$, and $n=2$ systems respectively can be found by

$$
\frac{e^{k_{2} x+\tau_{2} y+\omega_{2} t+\delta_{2}}}{1+A e^{\left(k_{1}+k_{2}\right) x+\left(\tau_{1}+\tau_{2}\right) y+\left(\omega_{1}+\omega_{2}\right) t+\delta_{1}+\delta_{2}}}=\frac{\sigma e^{\epsilon_{1} \bar{k}_{1} x+\epsilon_{2} \bar{\tau}_{1} y+\epsilon_{3} \bar{\omega}_{1} t+\bar{\delta}_{1}}}{1+\bar{A} e^{\epsilon_{1}\left(\bar{k}_{1}+\bar{k}_{2}\right) x+\epsilon_{2}\left(\bar{\tau}_{1}+\bar{\tau}_{2}\right) y+\epsilon_{3}\left(\bar{\omega}_{1}+\bar{\omega}_{2}\right) t+\bar{\delta}_{1}+\bar{\delta}_{2}}},
$$

where $A=-\frac{1}{\left(k_{1}+k_{2}\right)^{2}}$ and $\tau_{i}, i=1,2$ satisfy the dispersion relations given for each case $n=0,1,2$.

By applying the Type 1 approach, we obtain

$$
\text { 1) } k_{2}=\epsilon_{1} \bar{k}_{1}, \text { 2) } \omega_{2}=\epsilon_{3} \bar{\omega}_{1}, \text { 3) } e^{\delta_{2}}=\sigma e^{\bar{\delta}_{1}} \text {. }
$$

Using these constraints besides the conditions (5.6), (5.9), and (5.12) in the dispersion relations of the cases $n=0,1,2$ we get $\tau_{2}=\epsilon_{2} \bar{\tau}_{1}$.

Thus one-soliton solutions of the reduced Eqs. (5.5), (5.8), and (5.11) are given by

$$
p(x, y, t)=\frac{e^{k_{1} x+\tau_{1} y+\omega_{1} t+\delta_{1}}}{1-\frac{\sigma}{\left(k_{1}+\epsilon_{1} \bar{k}_{1}\right)^{2}} e^{\left(k_{1}+\epsilon_{1} \bar{k}_{1}\right) x+\left(\tau_{1}+\epsilon_{2} \bar{\tau}_{1}\right) y+\left(\omega_{1}+\epsilon_{3} \bar{\omega}_{1}\right) t+\delta_{1}+\bar{\delta}_{1}},}
$$

with the corresponding dispersion relations $\tau_{1}=\frac{1}{b}\left(\frac{1}{2} k_{1} \omega_{1}-a k_{1}\right), \tau_{1}=\frac{1}{b}\left(\frac{1}{2} k_{1} \omega_{1}-\frac{a}{2} k_{1}^{2}\right)$, and $\tau_{1}=\frac{1}{b}\left(\frac{1}{2} k_{1} \omega_{1}-\frac{a}{4} k_{1}^{3}\right)$ given respectively. It is clear that there are finite and singular solutions (7.32) depending on the parameters of the solutions.

Note that since there are 21 nonlocal reduced equations by the reduction formula $q(x, y, t)=\sigma \bar{p}\left(\epsilon_{1} x, \epsilon_{2} y, \epsilon_{3} t\right)$ for $n=$ $0,1,2$ let us only consider $y$-reflection that is when $\left(\epsilon_{1}, \epsilon_{2}, \epsilon_{3}\right)=(1,-1,1)$ as an example. Let $\sigma=-\left(k_{1}+\bar{k}_{1}\right)^{2} e^{\mu}, \mu$ is a real constant. Then one-soliton solutions of the nonlocal equations:

$$
(n=0), \quad b p_{y}(x, y, t)=\frac{1}{2} p_{t x}(x, y, t)-a p_{x}(x, y, t)-\sigma p(x, y, t) D^{-1}(p(x, y, t) \bar{p}(x,-y, t))_{t},
$$

where $a$ is a pure imaginary, $b$ is a real number,

$$
\begin{aligned}
(n=1), \quad b p_{y}(x, y, t)= & \frac{1}{2} p_{t x}(x, y, t)-\frac{a}{2} p_{x x}(x, y, t)+a \sigma p^{2}(x, y, t) \bar{p}(x,-y, t) \\
& -\sigma p(x, y, t) D^{-1}(p(x, y, t) \bar{p}(x,-y, t))_{t},
\end{aligned}
$$

where $a$ and $b$ are real numbers,

$$
\begin{aligned}
(n=2), \quad b p_{y}(x, y, t)= & \frac{1}{2} p_{t x}(x, y, t)-\frac{a}{4} p_{x x x}(x, y, t)+\frac{3 a}{2} \sigma p(x, y, t) \bar{p}(x,-y, t) p_{x}(x, y, t) \\
& -\sigma p(x, y, t) D^{-1}(p(x, y, t) \bar{p}(x,-y, t))_{t},
\end{aligned}
$$

where $a$ is a pure imaginary, $b$ is a real number, become

$$
p(x, y, t)=\frac{e^{\phi}}{2 \cosh (\theta)},
$$

where

$$
\begin{aligned}
& \left.\phi=\frac{1}{2}\left[\left(k_{1}-\bar{k}_{1}\right) x+\left(\tau_{1}+\bar{\tau}_{1}\right) y+\left(\omega_{1}-\bar{\omega}_{1}\right) t+\left(\delta_{1}-\bar{\delta}_{1}-\mu\right)\right)\right], \\
& \left.\theta=\frac{1}{2}\left[\left(k_{1}+\bar{k}_{1}\right) x+\left(\tau_{1}-\bar{\tau}_{1}\right) y+\left(\omega_{1}+\bar{\omega}_{1}\right) t+\left(\delta_{1}+\bar{\delta}_{1}-\mu\right)\right)\right] .
\end{aligned}
$$

The solution (7.36) is finite if $\tau_{1}-\bar{\tau}_{1} \in \mathbb{R}$ which happens when $\tau_{1} \in \mathbb{R}$. In addition to that it is bounded if $k_{1}-\bar{k}_{1}=0, \tau_{1}+$ $\bar{\tau}_{1}=2 \tau_{1}=0$, and $\omega_{1}-\bar{\omega}_{1} \leq 0$ for $t \geq 0$. This occurs only when $k_{1} \in \mathbb{R}$ and $\tau_{1}=0$. But taking $\tau_{1}=0$ reduces the dimension of the solution from $2+1$ to $1+1$.

\section{Conclusion}

In this work we obtained a new negative AKNS hierarchy denoted by AKNS $(-n)$ for $n=0,1,2, \ldots$ in $2+1$ dimensions. We obtained the Hirota bilinear forms of these systems and found one- and two-soliton solutions for $n=0,1,2$. We then found all possible local and nonlocal reductions of these systems. Using the constraint equations among the dynamical variables for $n=0,1,2$ we found 3 new local and 27 new nonlocal reduced equations in $2+1$ dimensions. These new nonlocal equations contain two different types of nonlocality. They contain terms with $D^{-1}$ (integro-differential equations) and terms $p\left(\epsilon_{1} x, \epsilon_{2} y, \epsilon_{3} t\right)$ (mirror symmetric terms) where $\epsilon_{1}^{2}=\epsilon_{2}^{2}=\epsilon_{3}^{2}=1$. From the one-soliton solutions of the negative AKNS system of equations we obtained one-soliton solutions of the local and nonlocal reduced equations. Among all these one-soliton solutions there are solutions which develop singularities in a finite time and there are also solutions which are finite and bounded depending on the parameters of the solutions. 


\section{Acknowledgment}

\section{This work is partially supported by the Scientific and Technological Research Council of Turkey (TÜBiTAK).}

\section{References}

[1] Gürses M, Pekcan A. 2+1 KdV(n) equations. J Math Phys 2011;52:083516. arXiv: 1102.5230v2[nlin.SI] [Preprint]. 2011 [cited 2011 Mar 3]: [15p.]. Available from: https://arxiv.org/abs/1102.5230.

[2] Karasu-Kalkanli A, Karasu A, Sakovich A, Sakovich S, Turhan R. A new integrable generalization of the Korteweg-de Vries equation. J Math Phys 2008;49:073516. arXiv: 0708.3247[nlin.SI] [Preprint]. 2007 [cited 2007 Aug 23]: [13p.]. Available from: https://arxiv.org/abs/0708.3247.

[3] Qiao Z. Finite-dimensional integrable system and nonlinear evolution equations (English). Beijing, PR China: Chinese National Higher Education Press; 2001 .

[4] Oiao Z. A general approach for getting the commutator representations of the hierarchies of nonlinear evolution equations. Phys Lett A 1994:195:319.

[5] Qiao Z. Generalized structure of lax representations for nonlinear evolution equation. Appl Math Mech 1997;18:671.

[6] Ablowitz MJ, Musslimani ZH. Integrable nonlocal nonlinear Schrödinger equation. Phys Rev Lett 2013;110:064105.

[7] Ablowitz MJ, Musslimani ZH. Inverse scattering transform for the integrable nonlocal nonlinear Schrödinger equation. Nonlinearity 2016;29:915.

[8] Ablowitz MJ, Musslimani ZH. Integrable nonlocal nonlinear equations. Stud Appl Math 2016;139(1):7.

[9] Ablowitz MJ, Kaup DJ, Newell AC, Segur H. The inverse scattering transform-Fourier analysis for nonlinear problems. Stud Appl Math 1974;53(4):249.

[10] Sakkaravarthi K, Kanna T. Bright solitons in coherently coupled nonlinear Schrödinger equations with alternate signs of nonlinearities. J Math Phys 2013;54:013701

[11] Gerdjikov VS, Saxena A. Complete integrability of nonlocal nonlinear Schrödinger equation. J Math Phys 2017:58(1):013502.

[12] Sinha D, Ghosh PK. Integrable nonlocal vector nonlinear Schrödinger equation with self-induced parity-time symmetric potential. Phys Lett A 2017;381:124.

[13] Gerdjikov VS, Grahovski DG, Ivanov RI. On integrable wave interactions and lax pairs on symmetric spaces. Wave Motion 2017;71:53.

[14] Gerdjikov VS, Grahovski DG, Ivanov RI. On the n-wave equations with PT symmetry. Theor Math Phys 2016;188(3):1305.

[15] Gerdjikov VS. On nonlocal models of Kulish-Sklyanin type and generalized fourier transforms. Stud Comput Int 2017;681:37.

[16] Khare A, Saxena A. Periodic and hyperbolic soliton solutions of a number of nonlocal nonlinear equations. J Math Phys 2015;56:032104.

[17] Li M, Xu T. Dark and antidark soliton interactions in the nonlocal nonlinear Schrödinger equation with the self-induced parity-time-symmetric potential. Phys Rev E 2015;91:033202.

[18] Huang X, King L. Soliton solutions for the nonlocal nonlinear Schrödinger equation. Eur Phys J Plus 2016;131:148.

[19] Wen XY, Yan Z, Yang Y. Dynamics of higher-order rational solitons for the nonlocal nonlinear Schrödinger equation with the self-induced paritytime-symmetric potential. Chaos 2015;26:063123.

[20] Caudrelier V. Interplay between the inverse scattering method and Fokas's unified transform with an application. Stud Appl Math 2017;140:3.

[21] Yang J. General n-solitons and their dynamics in several nonlocal nonlinear Schrödinger equations. arXiv:1712.01181v1 [nlin.SI] [Preprint]. 2017 cited[2017 Dec 4]: [11p.]. Available from: https://arxiv.org/abs/1712.01181.

[22] Feng BF, Luo XD, Ablowitz MJ, Musslimani ZH. General soliton solution to a nonlocal nonlinear Schrödinger equation with zero and nonzero boundary conditions. arXiv:1712.09172 [nlin.SI] [Preprint]. 2017 cited[2017 Dec 26]: [26p.]. Available from: https://arxiv.org/abs/1712.09172.

[23] Iwao M, Hirota R. Soliton solutions of a coupled modified KdV equations. J Phys Soc Japan 1997;66(3):577.

[24] Ma LY, Shen SF, Zhu ZN. Soliton solution and gauge equivalence for an integrable nonlocal complex modified Korteweg-de Vries equation. J Math Phys 2017;58:103501

[25] Ji JL, Zhu ZN. On a nonlocal modified Korteweg-de Vries equation: integrability, darboux transformation and soliton solutions. Commun Non Sci Numer Simul 2017;42:699

[26] Ji JL, Zhu ZN. Soliton solutions of an integrable nonlocal modified Korteweg-de Vries equation through inverse scattering transform. J Math An and App 2017;453:973.

[27] Yang B, Yang J. Transformations between nonlocal and local integrable equations. Stud Appl Math 2017:140:178.

[28] Chen K, Deng X, Lou S, Zhang D. Solutions of local and nonlocal equations reduced from the AKNS hierarchy. arXiv:1710.10479v2 [nlin.SI] [Preprint]. 2017 [cited 2017 Nov 12]: [26p.]. Available from: https://arxiv.org/abs/1710.10479.

[29] Fokas AS. Integrable multidimensional versions of the nonlocal Schrödinger equation. Nonlinearity 2016;29:319.

[30] Cao Y, He J, Rao J. Semi-rational solutions for the (2+1)-dimensional nonlocal Fokas system. Appl Math Lett 2018;80:27. arXiv: 1712.10013v2[nlin.SI] [Preprint]. 2018 cited[2018 Jan 9]: [7p.]. Available from: https://arxiv.org/1712.10013.

[31] Cao Y, Malomed BA, He J. Two (2+1)-dimensional integrable nonlocal nonlinear Schrödinger equations: breather, rational and semi-rational solutions. Chaos Solitons Fractals 2018;114:99.

[32] Gürses M, Pekcan A. Nonlocal nonlinear Schrödinger equations and their soliton solutions. J Math Phys 2018;59:051501. arXiv: 1707.07610v1[nlin.SI] [Preprint]. 2017 [cited 2017 Jul 24]: [30p.]. Available from: https://arxiv.org/abs/1707.07610.

[33] Gürses M, Pekcan A. Nonlocal nonlinear modified KdV equations and their soliton solutions. Commun Nonlinear Sci Numer Simul 2019;67:427. arXiv: 1711.01588v1[nlin.SI] [Preprint]. 2017 [cited 2017 Nov 5]: [52p.]. Available from: https://arxiv.org/abs/1711.01588.

[34] Gürses M. Nonlocal Fordy-Kulish equations on symmetric spaces. Phys Lett A 2017;381:1791.

[35] Gürses M. Nonlocal super integrable equations. arXiv:1704.01273 [nlin.SI] [Preprint]. 2017 cited[2017 Apr 5]: [8p.]. Available from: https://arxiv.org/ 1704.01273.

[36] Gürses M, Pekcan A. Integrable nonlocal reductions. 3rd international conference on symmetries, differential equations and applications 14-17 august; 2017. arXiv: 1805.01695[nlin.SI] [Preprint]. 2018 [cited ]: [24 p.]. Available from: https://arxiv.org/1805.01695.

[37] Bogoyavlenski OI. Breaking solitons IV. Math USSR Izv 1991;37(3):475.

[38] Bogoyavlenski OI. Breaking solitons in 2+1-dimensional integrable equations. Russ Math Surv 1990;45(4):1.

[39] Strachan IAB. Wave solutions of a (2+1)-dimensional generalization of the nonlinear Schrödinger equation. Inverse Probl 1992;8:L21.

[40] Liu YK, Li B. Rogue waves in the $(2+1)$-dimensional nonlinear Schrödinger equation with a parity-time symmetric potential. Chin Phys Lett 2017;34(1):010202 